\title{
MODELADO DINÁMICO Y SIMULACIÓN DE UN ROBOT TREPADOR TIPO SERIE CON 4 GRADOS DE LIBERTAD
}

\author{
Adrián Peidró, Alba Hortal, Arturo Gil, José María Marín, David Úbeda, Óscar Reinoso \\ Departamento de Ingeniería de Sistemas y Automática \\ Universidad Miguel Hernández, Avenida de la Universidad s/n, 03202 Elche (Alicante), España \\ \{adrian.peidro, alba.hortal, arturo.gil, jmarin, ubeda, o.reinoso\}@goumh.umh.es
}

\section{Resumen}

Este artículo presenta el modelado dinámico completo de un robot trepador con arquitectura serie y 4 grados de libertad. En primer lugar se obtiene el modelo cinemático del robot, modelándolo como un sistema articulado libre en el espacio. A continuación, se obtiene su modelo dinámico inverso empleando las ecuaciones de Lagrange. El modelo obtenido permite resolver la dinámica inversa del robot trepador estudiado independientemente de cuál de sus garras se fije al entorno o de cuál sea su postura en dicho entorno. El modelo obtenido ha sido implementado en una interfaz gráfica de usuario que permite simular la cinemática y la dinámica inversa del robot estudiado.

Palabras Clave: Cinemática, Dinámica inversa, Lagrange, Robot trepador, Simulador.

\section{INTRODUCCIÓN}

Este artículo presenta el análisis dinámico inverso del robot trepador mostrado en la Figura 1. Este robot pertenece a una clase de robots trepadores de estructuras formados por dos garras unidas por una cadena cinemática con arquitectura serie, paralela o híbrida. Estos robots se denominan de tipo "paso-apaso" [9] debido a su modo de locomoción: para moverse por una estructura, fijan una garra a la misma y utilizan la cadena cinemática para posicionar la otra garra en el siguiente punto de sujeción. A continuación, fijan esta última garra y liberan la primera, repitiendo el proceso. Estos robots disfrutan de una elevada movilidad para explorar estructuras, pero tienen como inconvenientes un peso elevado y una alta complejidad.

Durante las últimas dos décadas, numerosos autores han diseñado multitud de robots trepadores paso-apaso, con arquitecturas muy variadas. Por ejemplo, se han propuesto robots de arquitectura serie con 6 [2], 4 [9], 5 [4] y 8 [7] grados de libertad. También se ha diseñado un robot trepador basado en la plataforma paralela de Gough-Stewart [1]. Finalmente, otros autores han propuesto arquitecturas híbridas con 4 [10], 12 [3] y 10 [6] grados de libertad.

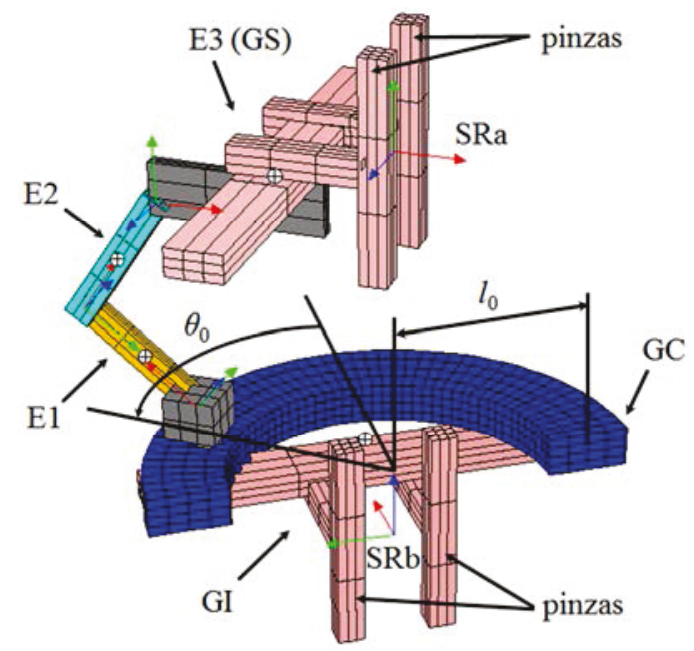

Figura 1: Robot trepador propuesto en [9]

Un aspecto clave en el diseño de robots trepadores de tipo paso-a-paso es el sistema de adhesión mediante el que el robot se sujeta a la estructura. Dicha adhesión puede ser de tipo mecánico (garras), magnético, por vacío, químico, etc., y su diseño requiere el análisis dinámico del robot.

En este artículo se obtiene el modelo dinámico del robot trepador 3DCLIMBER propuesto por Tavakoli et al. [9], mostrado en la Figura 1. El objetivo del análisis presentado aquí es estudiar los requerimientos de los actuadores de dicho robot, así como analizar las fuerzas y pares de sujeción en las garras del robot, para poder comparar en el futuro dichos parámetros con los correspondientes a los de otros robots trepadores. Aunque la dinámica del robot 3DCLIMBER ya fue estudiada en [8], el modelo allí presentado únicamente consideraba la dinámica plana de la cadena serie $3 \mathrm{R}$ del robot, asumiendo además que el robot trepa por un poste vertical paralelo a la aceleración de la gravedad. 
En cambio, en este artículo presentamos un análisis dinámico más general y completo del mencionado robot, en el que se modela el robot como un mecanismo articulado libre en el espacio, considerando todos sus grados de libertad y sin fijar a priori ninguna de sus garras a la estructura. De esta forma, el modelo presentado en este artículo permite resolver la dinámica inversa del mencionado robot independientemente de cuál de sus garras se fije a la estructura, sea cual sea la postura del robot o la posición de la estructura en la que se encuentre sujeto el mismo. El modelo obtenido en este artículo, además, ha sido implementado en una intuitiva interfaz gráfica de usuario que permite simular la cinemática y la dinámica del robot.

Este artículo está organizado como sigue. En la sección 2 se analiza la cinemática del robot trepador de la Figura 1. En la sección 3 se modela la dinámica de dicho robot mediante las ecuaciones de Lagrange. La sección 4 presenta una herramienta desarrollada que permite simular la dinámica del robot estudiado. Finalmente, la sección 5 concluye este artículo.

\section{CINEMÁTICA DEL ROBOT}

En esta sección se describe la arquitectura del robot trepador paso-a-paso propuesto por Tavakoli et al. [9], y se obtiene su modelo cinemático, que será necesario para construir posteriormente el modelo dinámico de dicho robot.

El robot trepador propuesto en [9] consta de dos garras unidas por una cadena cinemática de tipo serie con 4 grados de libertad, como se muestra en la Figura 1. La garra inferior (GI) tiene una guía circular (GC) sobre la que desliza una cadena serie plana de tipo $3 \mathrm{R}$, siendo $\theta_{0}$ el ángulo que forman el plano de simetría de la garra inferior y el plano de movimiento de la cadena $3 \mathrm{R}$ plana. La cadena plana 3R consta de tres eslabones E1, E2 y E3, conectados en serie a través de articulaciones de revolución con ángulos relativos $\theta_{1}, \theta_{2}$ y $\theta_{3}$, según muestra la Figura 2. El eslabón E3 coincide con la garra superior (GS).

Para analizar la cinemática y la dinámica de este robot se han definido varios sistemas de referencia solidarios a distintos cuerpos del mismo. Como es habitual, en este artículo los ejes $X, Y$ y $Z$ de los sistemas de referencia se representan mediante los colores rojo, verde y azul, respectivamente. El sistema de referencia $\mathrm{SRb}$ es solidario a la garra inferior. El origen de dicho sistema de referencia se encuentra en el punto medio entre las pinzas de la garra inferior, con los ejes orientados según se muestra en la Figura 1 (los ejes $X$ y $Z$ del sistema $\mathrm{SRb}$ definen el plano de simetría de la garra inferior, produciéndose la rotación $\theta_{0}$ alrededor del eje $Z$ ).

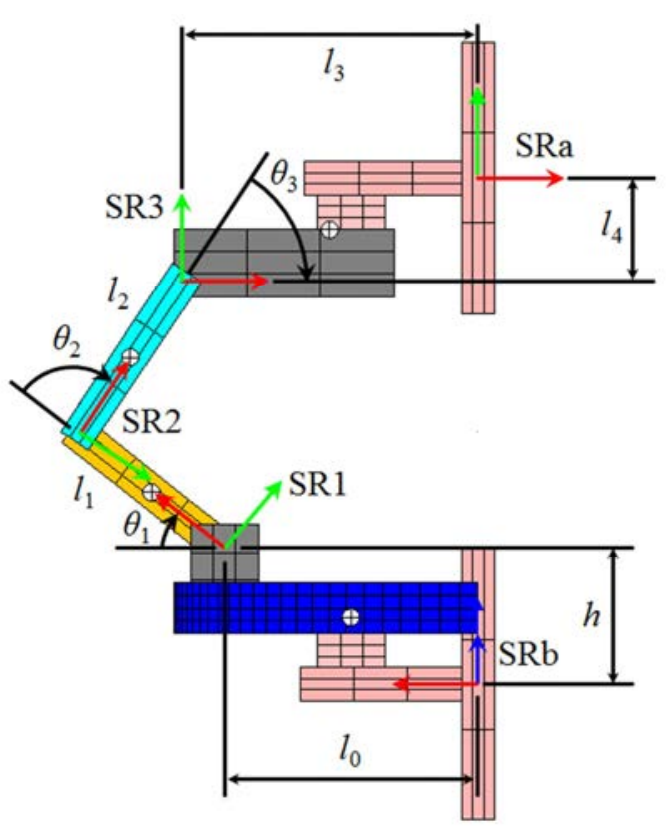

Figura 2: Cadena serie 3R plana del robot trepador

Por otra parte, otro sistema de referencia SR1 es solidario al eslabón E1 de la cadena plana 3R. Como muestra la Figura 2, el origen del sistema SR1 se encuentra sobre el eje de la rotación $\theta_{1}$, con su eje $X$ a lo largo del eslabón, su eje $Z$ coincidente con el eje de rotación de la articulación $\theta_{1}$, y su eje $Y$ escogido para formar un sistema dextrógiro. Según las Figuras 1 y 2, la transformación ${ }^{\mathrm{SRb}} \mathbf{T}_{\mathrm{SR} 1}$ entre los dos sistemas de referencia SRb y SR1 puede obtenerse multiplicando las siguientes cinco matrices de transformación homogénea:

$$
\begin{aligned}
&{ }^{\mathrm{SRb}} \mathbf{T}_{\mathrm{SR} 1}= {\left[\begin{array}{llll}
1 & 0 & 0 & 0 \\
0 & 1 & 0 & 0 \\
0 & 0 & 1 & h \\
0 & 0 & 0 & 1
\end{array}\right] \times\left[\begin{array}{cccc}
c_{0} & -s_{0} & 0 & 0 \\
s_{0} & c_{0} & 0 & 0 \\
0 & 0 & 1 & 0 \\
0 & 0 & 0 & 1
\end{array}\right] \times } \\
& {\left[\begin{array}{cccc}
1 & 0 & 0 & l_{0} \\
0 & 1 & 0 & 0 \\
0 & 0 & 1 & 0 \\
0 & 0 & 0 & 1
\end{array}\right] \times\left[\begin{array}{cccc}
1 & 0 & 0 & 0 \\
0 & \cos \left(\frac{\pi}{2}\right) & -\sin \left(\frac{\pi}{2}\right) & 0 \\
0 & \sin \left(\frac{\pi}{2}\right) & \cos \left(\frac{\pi}{2}\right) & 0 \\
0 & 0 & 0 & 1
\end{array}\right] \times } \\
& {\left[\begin{array}{cccc}
c_{1} & -s_{1} & 0 & 0 \\
s_{1} & c_{1} & 0 & 0 \\
0 & 0 & 1 & 0 \\
0 & 0 & 0 & 1
\end{array}\right] }
\end{aligned}
$$

donde $c_{i}=\cos \theta_{i}, s_{i}=\sin \theta_{i}, h$ es la distancia vertical entre los orígenes de los sistemas de referencia $\mathrm{SRb}$ y SR1 y $l_{0}$ es el radio medio de la guía circular. Según la Figura 2, la relación entre los sistemas de referencia SR1 y SR3 puede obtenerse como sigue: 


$$
\begin{aligned}
{ }^{\mathrm{SR} 1} \mathbf{T}_{\mathrm{SR} 3} & =\left[\begin{array}{llll}
1 & 0 & 0 & l_{1} \\
0 & 1 & 0 & 0 \\
0 & 0 & 1 & 0 \\
0 & 0 & 0 & 1
\end{array}\right] \times\left[\begin{array}{cccc}
c_{2} & -s_{2} & 0 & 0 \\
s_{2} & c_{2} & 0 & 0 \\
0 & 0 & 1 & 0 \\
0 & 0 & 0 & 1
\end{array}\right] \times \\
& {\left[\begin{array}{llll}
1 & 0 & 0 & l_{2} \\
0 & 1 & 0 & 0 \\
0 & 0 & 1 & 0 \\
0 & 0 & 0 & 1
\end{array}\right] \times\left[\begin{array}{cccc}
c_{3} & -s_{3} & 0 & 0 \\
s_{3} & c_{3} & 0 & 0 \\
0 & 0 & 1 & 0 \\
0 & 0 & 0 & 1
\end{array}\right] \times } \\
& {\left[\begin{array}{cccc}
1 & 0 & 0 & 0 \\
0 & \cos (\pi) & -\sin (\pi) & 0 \\
0 & \sin (\pi) & \cos (\pi) & 0 \\
0 & 0 & 0 & 1
\end{array}\right] }
\end{aligned}
$$

donde $l_{1}$ y $l_{2}$ son las longitudes de los eslabones E1 y E2, respectivamente. Finalmente, la relación entre el sistema SR3 y el sistema SRa viene dada por la siguiente transformación:

$$
{ }^{\mathrm{SR} 3} \mathbf{T}_{\mathrm{SRa}}=\left[\begin{array}{cccc}
1 & 0 & 0 & l_{3} \\
0 & 1 & 0 & l_{4} \\
0 & 0 & 1 & 0 \\
0 & 0 & 0 & 1
\end{array}\right]
$$

donde $l_{3}$ y $l_{4}$ son dimensiones de la garra superior del robot (ver Figura 2). Al igual que el sistema SR3, el sistema SRa es solidario a la garra superior, su origen está situado en el punto medio entre las pinzas de dicha garra, y su orientación con respecto a dicha garra es la mostrada en la Figura 1. Multiplicando todas las matrices anteriores se obtiene la transformación homogénea completa del robot, que proporciona la posición y orientación de la garra superior (sistema de referencia SRa) con respecto a la garra inferior (sistema de referencia $\mathrm{SRb}$ ):

$$
{ }^{\mathrm{SRb}} \mathbf{T}_{\mathrm{SRa}}={ }^{\mathrm{SRb}} \mathbf{T}_{\mathrm{SR} 1}{ }^{\mathrm{SR} 1} \mathbf{T}_{\mathrm{SR} 3}{ }^{\mathrm{SR} 3} \mathbf{T}_{\mathrm{SRa}}
$$

Para realizar el modelado dinámico en la siguiente sección, será conveniente definir explícitamente variables que definan las posiciones y orientaciones absolutas de ambas garras, tanto de la inferior como de la superior. Con "posición y orientación absolutas" nos referimos a la posición y orientación de las garras con respecto a un sistema de referencia "mundo" (que denotaremos por $\mathrm{W}$ ), fijado en algún punto de la estructura a explorar por el robot. La posición absoluta de la garra inferior se codificará mediante la posición $\left(x_{b}, y_{b}, z_{b}\right)$ del origen del sistema de referencia $\mathrm{SRb}$ solidario a la misma, mientras que su orientación absoluta se codificará mediante los 3 ángulos ZXZ de Euler $\left(\alpha_{1 b}, \alpha_{2 b}, \alpha_{3 b}\right)$, que expresan la orientación del sistema $\mathrm{SRb}$ con respecto al sistema del mundo W. De forma similar, la posición absoluta de la garra superior se codificará mediante la posición $\left(x_{a}, y_{a}, z_{a}\right)$ del sistema SRa, mientras que su orientación absoluta se codificará mediante los 3 ángulos ZXZ de Euler $\left(\alpha_{1 a}, \alpha_{2 a}, \alpha_{3 a}\right)$. A partir de las variables anteriores, la posición y orientación absolutas de cualquiera de las garras se pueden codificar en una sola matriz de transformación homogénea con la siguiente forma:

$$
{ }^{\mathrm{W}} \mathbf{T}_{\mathrm{SR} j}=\left[\begin{array}{cccc} 
& & & x_{j} \\
& { }^{\mathrm{W}} \mathbf{R}_{\mathrm{SR} j} & & y_{j} \\
0 & 0 & 0 & 1
\end{array}\right]
$$

donde $j=a$ para la garra superior, mientras que $j=b$ para la garra inferior. ${ }^{\mathrm{W}} \mathbf{R}_{\mathrm{SR} j}$ es la matriz $3 \times 3 \mathrm{de}$ rotación del sistema $\mathrm{SRj}$ con respecto al mundo, correspondiente a la elección ZXZ de ángulos de Euler:

$$
\begin{aligned}
& \mathrm{w}_{\mathbf{R}_{\mathrm{SR} j}}=\left[\begin{array}{cc}
c_{\alpha_{1 j}} c_{\alpha_{3 j}}-s_{\alpha_{1 j}} c_{\alpha_{2 j}} s_{\alpha_{3 j}} & \cdots \\
s_{\alpha_{1 j}} c_{\alpha_{3 j}}+c_{\alpha_{1 j}} c_{\alpha_{2 j}} s_{\alpha_{3 j}} & \cdots \\
s_{\alpha_{2 j}} s_{\alpha_{3 j}} & \cdots
\end{array}\right. \\
& \cdots-c_{\alpha_{1 j}} s_{\alpha_{3 j}}-s_{\alpha_{1 j}} c_{\alpha_{2 j}} c_{\alpha_{3 j}} \quad s_{\alpha_{1 j}} s_{\alpha_{2 j}} \\
& \left.\begin{array}{ccc}
\cdots & -s_{\alpha_{1 j}} s_{\alpha_{3 j}}+c_{\alpha_{1 j}} c_{\alpha_{2 j}} c_{\alpha_{3 j}} & -c_{\alpha_{1 j}} s_{\alpha_{2 j}} \\
\cdots & s_{\alpha_{2 j}} c_{\alpha_{3 j}} & c_{\alpha_{2 j}}
\end{array}\right]
\end{aligned}
$$

Es evidente que las posiciones y orientaciones absolutas de ambas garras no son independientes, sino que están relacionadas a través de la cadena cinemática de 4 grados de libertad que las une. Por tanto, las matrices ${ }^{\mathrm{W}} \mathbf{T}_{\text {SRa }} \mathrm{y}{ }^{\mathrm{W}} \mathbf{T}_{\mathrm{SRb}}$ están relacionadas a través de la siguiente restricción:

$$
{ }^{\mathrm{W}_{\mathrm{SRa}}} \mathbf{T}^{\mathrm{W}_{\mathbf{S R b}}}{ }^{\mathrm{SRb}} \mathbf{T}_{\mathrm{SRa}}
$$

Donde la matriz ${ }^{\mathrm{SRb}} \mathbf{T}_{\mathrm{SRa}}$, definida en la ecuación (4), depende de las 4 articulaciones de la cadena cinemática completa (ángulos $\theta_{0}, \theta_{1}, \theta_{2}$ y $\theta_{3}$ ).

\subsection{RELACIONES DE VELOCIDAD}

La ecuación matricial (7) introduce restricciones entre las variables del problema, de manera que la posición $\left(x_{a}, y_{a}, z_{a}\right)$ y la orientación $\left(\alpha_{1 a}, \alpha_{2 a}, \alpha_{3 a}\right)$ de la garra superior del robot quedan ambas determinadas por la posición $\left(x_{b}, y_{b}, z_{b}\right)$ y la orientación $\left(\alpha_{1 b}, \alpha_{2 b}, \alpha_{3 b}\right)$ de la garra inferior, así como por los 4 ángulos de la cadena cinemática que conecta ambas garras $\left(\theta_{0}, \theta_{1}, \theta_{2}\right.$ y $\left.\theta_{3}\right)$. Esta relación también se da a nivel de velocidades: las derivadas de las anteriores 16 variables están relacionadas a través de determinadas ecuaciones a obtener seguidamente.

En primer lugar, se obtendrá la relación entre la velocidad lineal $\left(\dot{x}_{a}, \dot{y}_{a}, \dot{z}_{a}\right)$ de la garra superior y el resto de velocidades. Igualando los elementos $(1,4)$, $(2,4)$ y $(3,4)$ de las matrices del lado izquierdo y derecho de la ecuación (7) se obtienen las siguientes ecuaciones:

$$
\begin{aligned}
& x_{a}=f_{x_{a}}\left(x_{b}, y_{b}, z_{b}, \alpha_{1 b}, \alpha_{2 b}, \alpha_{3 b}, \theta_{0}, \theta_{1}, \theta_{2}, \theta_{3}\right) \\
& y_{a}=f_{y_{a}}\left(x_{b}, y_{b}, z_{b}, \alpha_{1 b}, \alpha_{2 b}, \alpha_{3 b}, \theta_{0}, \theta_{1}, \theta_{2}, \theta_{3}\right)
\end{aligned}
$$




$$
z_{a}=f_{z_{a}}\left(x_{b}, y_{b}, z_{b}, \alpha_{1 b}, \alpha_{2 b}, \alpha_{3 b}, \theta_{0}, \theta_{1}, \theta_{2}, \theta_{3}\right)
$$

donde $f_{x_{a}}, f_{y_{a}}$ y $f_{z_{a}}$ denotan funciones de las variables $\left\{x_{b}, y_{b}, z_{b}, \alpha_{1 b}, \alpha_{2 b}, \alpha_{3 b}, \theta_{0}, \theta_{1}, \theta_{2}, \theta_{3}\right\}$ (la expresión de dichas funciones no se incluye aquí debido a su elevado tamaño). Agrupando todos los términos al mismo lado de cada ecuación, las anteriores ecuaciones pueden escribirse como sigue:

$$
\left[\begin{array}{l}
\Gamma_{1} \\
\Gamma_{2} \\
\Gamma_{3}
\end{array}\right]=\left[\begin{array}{l}
0 \\
0 \\
0
\end{array}\right]
$$

donde $\Gamma_{1}=f_{x_{a}}-x_{a}, \Gamma_{2}=f_{y_{a}}-y_{a}$ y $\Gamma_{3}=f_{z_{a}}-z_{a}$. Derivando con respecto al tiempo la ecuación (11) se obtiene la siguiente ecuación de velocidades:

$$
\left[\begin{array}{lllll}
\frac{\partial \Gamma_{1}}{\partial x_{b}} & \frac{\partial \Gamma_{1}}{\partial y_{b}} & \cdots & \frac{\partial \Gamma_{1}}{\partial \alpha_{2 a}} & \frac{\partial \Gamma_{1}}{\partial \alpha_{3 a}} \\
\frac{\partial \Gamma_{2}}{\partial x_{b}} & \frac{\partial \Gamma_{2}}{\partial y_{b}} & \cdots & \frac{\partial \Gamma_{2}}{\partial \alpha_{2 a}} & \frac{\partial \Gamma_{2}}{\partial \alpha_{3 a}} \\
\frac{\partial \Gamma_{3}}{\partial x_{b}} & \frac{\partial \Gamma_{3}}{\partial y_{b}} & \cdots & \frac{\partial \Gamma_{3}}{\partial \alpha_{2 a}} & \frac{\partial \Gamma_{3}}{\partial \alpha_{3 a}}
\end{array}\right] \dot{\mathbf{q}}=\left[\begin{array}{l}
0 \\
0 \\
0
\end{array}\right]
$$

donde $\mathbf{q}=\left[x_{b}, y_{b}, z_{b}, \alpha_{1 b}, \alpha_{2 b}, \alpha_{3 b}, \theta_{0}, \theta_{1}, \theta_{2}, \theta_{3}\right.$, $\left.x_{a}, y_{a}, z_{a}, \alpha_{1 a}, \alpha_{2 a}, \alpha_{3 a}\right]^{T}$ (los puntos suspensivos en la ecuación (12) indican que deben escribirse todas las columnas de las parciales con respecto a todas las variables del vector $\mathbf{q}$, en el orden en que aparecen en q). Conocidas las velocidades lineal y angular de la garra inferior, y las velocidades articulares $\left(\dot{\theta}_{0}, \dot{\theta}_{1}, \dot{\theta}_{2}, \dot{\theta}_{3}\right)$, podría obtenerse la velocidad lineal $\left(\dot{x}_{a}, \dot{y}_{a}, \dot{z}_{a}\right)$ de la garra superior a partir de la anterior ecuación.

Para obtener la relación entre las velocidades $\left(\dot{\alpha}_{1 a}, \dot{\alpha}_{2 a}, \dot{\alpha}_{3 a}\right)$ y las velocidades del resto de variables angulares, calcularemos la velocidad angular $\boldsymbol{\Omega}_{a}$ de la garra superior por dos caminos distintos. Dado un sistema de referencia, con su orientación representada por una matriz de rotación $\mathbf{R}$, el vector de velocidad angular $\boldsymbol{\Omega}$ de dicho sistema de referencia puede obtenerse como [5]:

$$
\langle\boldsymbol{\Omega}\rangle=\dot{\mathbf{R}} \mathbf{R}^{T} \text {, donde: } \dot{\mathbf{R}}=\sum_{\psi=1}^{n}\left(\partial \mathbf{R} / \partial \alpha_{\psi}\right) \dot{\alpha}_{\psi}
$$

y donde $\langle\boldsymbol{\Omega}\rangle$ denota la matriz antisimétrica asociada al vector $\mathbf{\Omega}$. Según (13), si la matriz de rotación $\mathbf{R}$ depende de varias coordenadas angulares $\left\{\alpha_{1}, \alpha_{2}, \ldots, \alpha_{n}\right\}$, entonces el vector $\boldsymbol{\Omega}$ debe poder escribirse como combinación lineal de las velocidades $\dot{\alpha}_{\psi}$ de dichas coordenadas angulares (que son distintas, en general, a las componentes del vector de velocidad angular). Teniendo esto en cuenta y aplicando la ecuación (13) con la submatriz de rotación de la matriz ${ }^{\mathrm{W}} \mathbf{T}_{\mathrm{SRa}}$ del lado izquierdo de la ecuación (7), se obtiene el vector de velocidad angular $\boldsymbol{\Omega}_{a}$ de la garra superior:

$$
\boldsymbol{\Omega}_{a}=\left[\begin{array}{ccc}
0 & \cos \alpha_{1 a} & \sin \alpha_{1 a} \sin \alpha_{2 a} \\
0 & \sin \alpha_{1 a} & -\cos \alpha_{1 a} \sin \alpha_{2 a} \\
1 & 0 & \cos \alpha_{2 a}
\end{array}\right]\left[\begin{array}{c}
\dot{\alpha}_{1 a} \\
\dot{\alpha}_{2 a} \\
\dot{\alpha}_{3 a}
\end{array}\right]
$$

Por otro lado, el vector $\boldsymbol{\Omega}_{a}$ también puede obtenerse aplicando la ecuación (13) con la submatriz de rotación de la matriz $\left[{ }^{\mathrm{W}} \mathbf{T}_{\mathrm{SRb}}{ }^{\mathrm{SRb}} \mathbf{T}_{\mathrm{SRa}}\right]$, del lado derecho de la ecuación (7), lo cual proporciona una expresión de la siguiente forma, análoga a (14):

$$
\boldsymbol{\Omega}_{a}=\mathbf{H}\left[\dot{\alpha}_{1 b}, \dot{\alpha}_{2 b}, \dot{\alpha}_{3 b}, \dot{\theta}_{0}, \dot{\theta}_{1}, \dot{\theta}_{2}, \dot{\theta}_{3}\right]^{T}
$$

donde $\mathbf{H}$ es una matriz de tamaño $3 \times 7$, cuya expresión no se muestra debido a su elevado tamaño. Igualando el lado derecho de las ecuaciones (14) y (15), y agrupando todos los términos en el mismo lado de la ecuación resultante, se obtiene la siguiente ecuación, en la que los términos de la matriz de coeficientes (que son conocidos) se han denotado por las derivadas parciales con respecto a tres funciones $\Gamma_{4}, \Gamma_{5}$ y $\Gamma_{6}$, cuyas expresiones explícitas son desconocidas:

$$
\left[\begin{array}{lllll}
\frac{\partial \Gamma_{4}}{\partial x_{b}} & \frac{\partial \Gamma_{4}}{\partial y_{b}} & \cdots & \frac{\partial \Gamma_{4}}{\partial \alpha_{2 a}} & \frac{\partial \Gamma_{4}}{\partial \alpha_{3 a}} \\
\frac{\partial \Gamma_{5}}{\partial x_{b}} & \frac{\partial \Gamma_{5}}{\partial y_{b}} & \cdots & \frac{\partial \Gamma_{5}}{\partial \alpha_{2 a}} & \frac{\partial \Gamma_{5}}{\partial \alpha_{3 a}} \\
\frac{\partial \Gamma_{6}}{\partial x_{b}} & \frac{\partial \Gamma_{6}}{\partial y_{b}} & \cdots & \frac{\partial \Gamma_{6}}{\partial \alpha_{2 a}} & \frac{\partial \Gamma_{6}}{\partial \alpha_{3 a}}
\end{array}\right] \dot{\mathbf{q}}=\left[\begin{array}{l}
0 \\
0 \\
0
\end{array}\right]
$$

Conociendo las velocidades de las variables articulares $\left(\dot{\theta}_{0}, \dot{\theta}_{1}, \dot{\theta}_{2}, \dot{\theta}_{3}\right)$ y la velocidad de los ángulos de Euler de la garra inferior $\left(\dot{\alpha}_{1 b}, \dot{\alpha}_{2 b}, \dot{\alpha}_{3 b}\right)$, podrían despejarse de la anterior ecuación las velocidades de los ángulos de Euler de la garra superior $\left(\dot{\alpha}_{1 a}, \dot{\alpha}_{2 a}, \dot{\alpha}_{3 a}\right)$.

Cabe destacar que las anteriores ecuaciones de velocidad (12) y (16) pueden derivarse con respecto al tiempo para obtener las relaciones de aceleración, que permitirían determinar las aceleraciones de la garra superior $\left(\ddot{x}_{a}, \ddot{y}_{a}, \ddot{z}_{a}, \ddot{\alpha}_{1 a}, \ddot{\alpha}_{2 a}, \ddot{\alpha}_{3 a}\right)$ a partir de las aceleraciones de la garra inferior $\left(\ddot{x}_{b}, \ddot{y}_{b}, \ddot{z}_{b}, \ddot{\alpha}_{1 b}\right.$, $\left.\ddot{\alpha}_{2 b}, \ddot{\alpha}_{3 b}\right)$, de las aceleraciones articulares $\left(\ddot{\theta}_{0}, \ddot{\theta}_{1}, \ddot{\theta}_{2}\right.$, $\left.\ddot{\theta}_{3}\right)$, y de las velocidades de todas las 16 variables.

\section{MODELADO DINÁMICO}

En esta sección se obtiene el modelo dinámico del robot trepador estudiado en este artículo. El objetivo de obtener dicho modelo dinámico es, principalmente, resolver el problema dinámico inverso del robot: dado un estado de movimiento del robot (caracterizado por la posición y orientación de 
todos los cuerpos del robot, así como por sus velocidades y aceleraciones), deseamos obtener las fuerzas y los pares necesarios para lograr tal estado de movimiento.

El resultado del análisis de la dinámica inversa no debe ser únicamente el valor de los pares a aplicar en las cuatro articulaciones del robot por sus cuatro actuadores (pares en las articulaciones correspondientes a los giros $\theta_{0}, \theta_{1}, \theta_{2}$ y $\theta_{3}$ ), sino que también estamos especialmente interesados en conocer las fuerzas y pares de sujeción que debe proporcionar la garra fija del robot (sea la garra superior o la inferior) para mantenerlo sujeto a la estructura por la que trepa. Para realizar el modelado dinámico del robot trepador, en principio se considerará que éste se encuentra moviéndose libremente en el espacio, sin ninguna de sus dos garras sujetando la estructura. La sujeción de alguna de las garras a la estructura será una condición a imponer posteriormente.

La dinámica del robot estudiado se modelará mediante las ecuaciones de Lagrange para sistemas con restricciones, considerando que la ecuación (7) impone restricciones entre las variables cinemáticas. En un sistema mecánico con restricciones entre sus coordenadas generalizadas, cada una de las coordenadas generalizadas $q_{u}$ del sistema $(u=$ $1, \ldots, D)$ añade a la dinámica una ecuación como la siguiente [11]:

$$
F_{q_{u}}=\frac{d}{d t}\left(\frac{\partial L}{\partial \dot{q}_{u}}\right)-\frac{\partial L}{\partial q_{u}}-\sum_{r=1}^{C} \lambda_{r} \frac{\partial \Gamma_{r}}{\partial q_{u}}
$$

donde $L=K-V$ es el Lagrangiano del sistema mecánico, siendo $K$ la energía cinética total de dicho sistema y $V$ su energía potencial total. $F_{q_{u}}$ es la fuerza generalizada asociada a la coordenada generalizada $q_{u}$, de forma que $F_{q_{u}}$ será una fuerza si $q_{u}$ tiene unidades de longitud, o bien $F_{q_{u}}$ será un momento si $q_{u}$ tiene unidades angulares. $\lambda_{r}(r=$ $1, \ldots, C$ ) son los multiplicadores de Lagrange (siendo $C$ el número de restricciones) y $\Gamma_{r}$ es la $r$-ésima función de restricción.

Las coordenadas generalizadas a considerar en el modelo dinámico son las siguientes: $q_{u} \in Q=\left\{x_{b}\right.$, $y_{b}, z_{b}, \alpha_{1 b}, \alpha_{2 b}, \alpha_{3 b}, \theta_{0}, \theta_{1}, \theta_{2}, \theta_{3}, x_{a}, y_{a}, z_{a}, \alpha_{1 a}$, $\alpha_{2 a}, \alpha_{3 a}$. Por tanto, existirá una ecuación como (17) por cada una de estas 16 coordenadas.

En cuanto a los términos de derivadas parciales $\partial \Gamma_{r} / \partial q_{u}$ en la ecuación (17), que introducen fuerzas de restricción, en el modelo a plantear existen $C=6$ restricciones entre las coordenadas generalizadas ( 3 de posición y 3 de orientación), y las derivadas parciales a emplear en la ecuación de Lagrange son las definidas en las ecuaciones de velocidad (12) y (16).

En las siguientes subsecciones se describe la obtención del resto de términos de la ecuación (17) para las distintas coordenadas generalizadas, así como la resolución de la ecuación matricial resultante para obtener la solución de la dinámica inversa para un determinado estado de movimiento del robot.

\subsection{CONSTRUCCIÓN DEL LAGRANGIANO}

Para obtener el Lagrangiano $L$ se precisa obtener la energía cinética y potencial de todos los cuerpos del robot: las 2 garras y los eslabones E1 y E2.

La energía potencial $V_{k}$ de cada cuerpo $k$ se puede obtener como $V_{k}=-m_{k} \mathbf{g}^{T} \mathbf{r}_{\mathrm{cm}, k}^{\mathrm{W}}$, donde $\mathbf{r}_{\mathrm{cm}, k}^{\mathrm{W}}$ es la posición absoluta (con respecto al mundo $\mathrm{W}$ ) del centro de masa del cuerpo $k, m_{k}$ es su masa y $\mathbf{g}=\left[g_{x}, g_{y}, g_{z}\right]^{T}$ es el vector de gravedad en ejes del sistema de referencia del mundo W. Se asume que, en cada uno de los 4 cuerpos del robot, la masa está distribuida de forma uniforme, resultando en las correspondientes posiciones de los centros de masa.

En cuanto a la energía cinética $K_{k}$ de cada cuerpo $k$, ésta comprende la energía de traslación del centro de masa y la energía de rotación alrededor del centro de masa. La velocidad lineal de cada centro de masa, necesaria para calcular la energía de traslación, se obtiene como la derivada temporal del vector $\mathbf{r}_{\mathrm{cm}, k}^{\mathrm{W}}$ de cada cuerpo. Para calcular la energía de rotación de cada cuerpo es preciso obtener su velocidad angular, que puede obtenerse de la ecuación (13), utilizando la matriz de rotación absoluta (con respecto al mundo W) ligada a dicho cuerpo.

Debido al gran tamaño de los términos involucrados (vectores de posición, velocidades, matrices de rotación, etc.), la expresión final del Lagrangiano $L$ es demasiado extensa y no se incluirá aquí.

\subsection{DERIVADAS DEL LAGRANGIANO}

Una vez se dispone del Lagrangiano $L$, es necesario calcular sus derivadas en la ecuación (17). En primer lugar, la regla de la cadena permite expresar la derivada temporal de la ecuación (17) como una suma de derivadas parciales de segundo orden multiplicadas por las velocidades y aceleraciones de todas las coordenadas generalizadas, ya que en general la derivada parcial $\partial L / \partial \dot{q}_{u}$ es función de dichas coordenadas y de sus velocidades:

$$
\frac{d}{d t}\left(\frac{\partial L}{\partial \dot{q}_{u}}\right)=\sum_{q_{w} \in Q}\left(\frac{\partial^{2} L}{\partial q_{w} \partial \dot{q}_{u}} \dot{q}_{w}+\frac{\partial^{2} L}{\partial \dot{q}_{w} \partial \dot{q}_{u}} \ddot{q}_{w}\right)
$$


De esta forma, todas las derivadas de $L$ a calcular en la ecuación (17) son ahora derivadas parciales de primer y segundo orden. Sin embargo, dado que la expresión de $L$ es bastante complicada y extensa, el cálculo simbólico de todas las derivadas parciales necesarias es inviable. Por tanto, como alternativa al cálculo simbólico de las derivadas parciales, realizaremos el cálculo numérico de dichas derivadas parciales aproximándolas por diferencias centrales, mejorando la precisión del resultado mediante el método de extrapolación de Richardson.

\subsection{RESOLUCIÓN DE LA DINÁMICA INVERSA}

Una vez se ha determinado cómo obtener los distintos términos de la ecuación de Lagrange (17), se particulariza dicha ecuación para cada una de las 16 coordenadas generalizadas $q_{u} \in Q=\left\{x_{b}, y_{b}, z_{b}\right.$, $\alpha_{1 b}, \alpha_{2 b}, \alpha_{3 b}, \theta_{0}, \theta_{1}, \theta_{2}, \theta_{3}, x_{a}, y_{a}, z_{a}, \alpha_{1 a}, \alpha_{2 a}$, $\left.\alpha_{3 a}\right\}$, obteniendo un sistema de 16 ecuaciones que puede escribirse matricialmente como sigue:

$$
\left[\begin{array}{c}
F_{x_{b}} \\
F_{y_{b}} \\
\vdots \\
F_{\alpha_{2 a}} \\
F_{\alpha_{3 a}}
\end{array}\right]=\left[\begin{array}{c}
\frac{d}{d t}\left(\frac{\partial L}{\partial \dot{x}_{b}}\right)-\frac{\partial L}{\partial x_{b}} \\
\frac{d}{d t}\left(\frac{\partial L}{\partial \dot{y}_{b}}\right)-\frac{\partial L}{\partial y_{b}} \\
\vdots \\
\frac{d}{d t}\left(\frac{\partial L}{\partial \dot{\alpha}_{2 a}}\right)-\frac{\partial L}{\partial \alpha_{2 a}} \\
\frac{d}{d t}\left(\frac{\partial L}{\partial \dot{\alpha}_{3 a}}\right)-\frac{\partial L}{\partial \alpha_{3 a}}
\end{array}\right]-
$$$$
\left[\begin{array}{cccccc}
\frac{\partial \Gamma_{1}}{\partial x_{b}} & \frac{\partial \Gamma_{2}}{\partial x_{b}} & \frac{\partial \Gamma_{3}}{\partial x_{b}} & \frac{\partial \Gamma_{4}}{\partial x_{b}} & \frac{\partial \Gamma_{5}}{\partial x_{b}} & \frac{\partial \Gamma_{6}}{\partial x_{b}} \\
\frac{\partial \Gamma_{1}}{\partial y_{b}} & \frac{\partial \Gamma_{2}}{\partial y_{b}} & \frac{\partial \Gamma_{3}}{\partial y_{b}} & \frac{\partial \Gamma_{4}}{\partial y_{b}} & \frac{\partial \Gamma_{5}}{\partial y_{b}} & \frac{\partial \Gamma_{6}}{\partial y_{b}} \\
\vdots & \vdots & \vdots & \vdots & \vdots & \vdots \\
\frac{\partial \Gamma_{1}}{\partial \alpha_{2 a}} & \frac{\partial \Gamma_{2}}{\partial \alpha_{2 a}} & \frac{\partial \Gamma_{3}}{\partial \alpha_{2 a}} & \frac{\partial \Gamma_{4}}{\partial \alpha_{2 a}} & \frac{\partial \Gamma_{5}}{\partial \alpha_{2 a}} & \frac{\partial \Gamma_{6}}{\partial \alpha_{2 a}} \\
\frac{\partial \Gamma_{1}}{\partial \alpha_{3 a}} & \frac{\partial \Gamma_{2}}{\partial \alpha_{3 a}} & \frac{\partial \Gamma_{3}}{\partial \alpha_{3 a}} & \frac{\partial \Gamma_{4}}{\partial \alpha_{3 a}} & \frac{\partial \Gamma_{5}}{\partial \alpha_{3 a}} & \frac{\partial \Gamma_{6}}{\partial \alpha_{3 a}}
\end{array}\right]\left[\begin{array}{l}
\lambda_{1} \\
\lambda_{2} \\
\lambda_{3} \\
\lambda_{4} \\
\lambda_{5} \\
\lambda_{6}
\end{array}\right]
$$

La resolución de la dinámica inversa proporciona las fuerzas generalizadas que son necesarias en cada uno de los grados de libertad del robot para lograr un determinado estado de movimiento del mismo, determinado por las 16 coordenadas generalizadas, las velocidades de éstas y sus aceleraciones. Conocido el estado de movimiento del robot, se conoce la matriz de derivadas del Lagrangiano (el primer vector columna del lado derecho de la ecuación (19)), así como la matriz de derivadas parciales $\partial \Gamma_{r} / \partial q_{u}$ del lado derecho de la ecuación (19). Por tanto, para la dinámica inversa, las únicas incógnitas de la ecuación (19) son las 16 fuerzas generalizadas del lado izquierdo de la ecuación, así como los 6 multiplicadores de Lagrange del lado derecho. Además, todas estas incógnitas aparecen linealmente en el sistema a resolver. El problema es que la ecuación matricial (19) únicamente provee 16 ecuaciones escalares para determinar $16+6$ incógnitas, por lo que sobran incógnitas. Este problema desaparece cuando fijamos una de las garras del robot a la estructura y dejamos la otra garra libre, como se describe a continuación.

Cuando se fija la garra inferior a la estructura, las fuerzas externas sobre la garra superior son nulas ya que ésta se encuentra libre (no se cuenta la gravedad, pues ya ha sido contabilizada a través del Lagrangiano). Por tanto: $F_{x_{a}}=F_{y_{a}}=F_{z_{a}}=F_{\alpha_{1 a}}=F_{\alpha_{2 a}}$ $=F_{\alpha_{3 a}}=0$. En tal caso, el sistema (19) únicamente tiene 16 incógnitas, que pueden resolverse como sigue: en primer lugar, de las 6 ecuaciones correspondientes a las coordenadas de la garra superior (que son las últimas 6 ecuaciones de (19), en las que el lado izquierdo es ahora nulo), se resuelven los 6 multiplicadores de Lagrange $\left(\lambda_{1}, \ldots, \lambda_{6}\right)$. A continuación, se sustituyen dichos multiplicadores de Lagrange en las restantes 10 ecuaciones (que son las 10 primeras ecuaciones de (19)). Esto permite obtener el resto de fuerzas generalizadas: los pares de las 4 articulaciones del robot $\left(F_{\theta_{0}}, F_{\theta_{1}}, F_{\theta_{2}}, F_{\theta_{3}}\right)$, las fuerzas de sujeción $\left(F_{x_{b}}, F_{y_{b}}, F_{z_{b}}\right)$ en la garra inferior, que está sujeta a la estructura, y los momentos de sujeción en dicha garra $\left(F_{\alpha_{1 b}}, F_{\alpha_{2 b}}, F_{\alpha_{3 b}}\right)$.

Similarmente, si se fija la garra superior y se deja libre la inferior, entonces: $F_{x_{b}}=F_{y_{b}}=F_{z_{b}}=F_{\alpha_{1 b}}=$ $F_{\alpha_{2 b}}=F_{\alpha_{3 b}}=0$. En tal caso, de las primeras 6 ecuaciones de (19) se obtendrían los 6 multiplicadores de Lagrange, cuyo valor se sustituiría en las últimas 10 ecuaciones de (19) para obtener los pares de las 4 articulaciones del robot, las fuerzas de sujeción $\left(F_{x_{a}}\right.$, $\left.F_{y_{a}}, F_{z_{a}}\right)$ en la garra superior, y los momentos de sujeción en dicha garra $\left(F_{\alpha_{1 a}}, F_{\alpha_{2 a}}, F_{\alpha_{3 a}}\right)$.

De esta forma, mediante un único modelo dinámico se puede resolver el problema dinámico inverso del robot estudiado, independientemente de qué garra se encuentre sujeta a la estructura.

\subsection{PARES ASOCIADOS A LOS ÁNGULOS DE EULER}

La interpretación de las fuerzas generalizadas asociadas a las variables $\left(\theta_{0}, \theta_{1}, \theta_{2}, \theta_{3}\right)$ es sencilla: dichas fuerzas generalizadas son los momentos necesarios en las 4 articulaciones del robot (momentos que serían generados por los actuadores).

También es fácil interpretar las fuerzas generalizadas asociadas a las variables $x_{j}, y_{j}$ y $z_{j}(j \in\{a, b\})$ de la garra fija: son las fuerzas que debe proveer la garra para mantener el robot adherido a la estructura, en la dirección de los ejes $X, Y$ y $Z$, respectivamente. 
La interpretación de las fuerzas generalizadas asociadas a los ángulos de Euler $\left(\alpha_{1 j}, \alpha_{2 j}, \alpha_{3 j}\right)$ de la garra fija no es tan intuitiva, ya que las rotaciones ZXZ de Euler se producen de forma sucesiva alrededor de ejes móviles. Por tanto, dichas fuerzas generalizadas no deben interpretarse como momentos de sujeción alrededor de los ejes Cartesianos del sistema de referencia del mundo (W). Sin embargo, es posible obtener el momento de sujeción $\mathbf{M}_{j}$ alrededor de los ejes Cartesianos del mundo, que es fácil de interpretar, a partir de los pares de Euler. Para ello se puede emplear la siguiente relación, análoga a la relación (14) entre la velocidad angular y las velocidades de los ángulos de Euler:

$$
\mathbf{M}_{j}=\left[\begin{array}{ccc}
0 & \cos \alpha_{1 j} & \sin \alpha_{1 j} \sin \alpha_{2 j} \\
0 & \sin \alpha_{1 j} & -\cos \alpha_{1 j} \sin \alpha_{2 j} \\
1 & 0 & \cos \alpha_{2 j}
\end{array}\right]^{-T}\left[\begin{array}{c}
F_{\alpha_{1 j}} \\
F_{\alpha_{2 j}} \\
F_{\alpha_{3 j}}
\end{array}\right]
$$

\section{SIMULACIÓN Y EJEMPLOS}

Esta sección presenta una herramienta de simulación que se ha desarrollado para poder simular y analizar la dinámica de los movimientos del robot trepador estudiado en este artículo en una estructura tridimensional. La herramienta puede descargarse de http://arvc.umh.es/parola/serial4climber.html (podría ser necesario actualizar Java para ejecutarla). Entre otras funcionalidades, la herramienta implementa los modelos cinemático y dinámico inverso descritos en las secciones previas.

La herramienta desarrollada, mostrada en la Figura 3, consta de 4 ventanas. La ventana V1 muestra el robot trepando por una estructura 3D. La ventana V2 permite cambiar la posición de la garra fija $\mathrm{y}$ modificar la geometría del robot o de la estructura. La ventana V3 permite cambiar la garra que se sujeta a la estructura, y también permite simular la cinemática directa, modificando el valor de los ángulos $\theta_{0}, \theta_{1}, \theta_{2}$ y $\theta_{3}$. La ventana $\mathrm{V} 3$ también permite visualizar el espacio de trabajo o simular la cinemática inversa, introduciendo la posición deseada para la garra libre (ambos problemas han sido estudiados en [8]). Finalmente, la ventana V4 permite introducir las velocidades y aceleraciones de las distintas coordenadas generalizadas y simular la dinámica inversa, obteniendo y mostrando los momentos necesarios en las articulaciones del robot, así como las fuerzas y pares de sujeción necesarios en la garra fija, para conseguir el estado de movimiento introducido por el usuario.

A continuación se mostrarán dos ejemplos de la dinámica inversa del robot estudiado, resueltos mediante la herramienta de simulación presentada.

\subsection{EJEMPLOS}

Para los ejemplos, se asume que ambas garras tienen una masa de $1 \mathrm{~kg}$, mientras que los eslabones E1 y E2 tienen una masa de $0.5 \mathrm{~kg}$. La gravedad actúa en el sentido negativo del eje $Z: \mathbf{g}=[0,0,-9.81]^{T} \mathrm{~m} / \mathrm{s}^{2}$. La posición de $\mathrm{SRb}$ es $x_{b}=0, y_{b}=0, z_{b}=0$, mientras que su orientación es $\alpha_{1 b}=\alpha_{3 b}=0, \alpha_{2 b}=\pi / 4 \mathrm{rad}$. Los ángulos de las 4 articulaciones son (en radianes): $\theta_{0}=0, \theta_{1}=0.672, \theta_{2}=1.487$ y $\theta_{3}=0.982$. El robot se encuentra trepando por una barra a $45^{\circ}$, como se muestra en la Figura 4.

Para el primer ejemplo, el robot se encuentra parado en la configuración anterior (todas las velocidades y aceleraciones son nulas), con la garra inferior agarrando la estructura y la superior libre. La resolución de la dinámica inversa proporciona las siguientes fuerzas y momentos: $F_{x_{b}}=F_{y_{b}}=0, F_{z_{b}}=29.43$ $\mathrm{N}, \mathbf{M}_{b}=[-6.5,-7.1,0]^{T} \mathrm{~N} \cdot \mathrm{m}, F_{\theta_{0}}=3.9 \mathrm{~N} \cdot \mathrm{m}, F_{\theta_{1}}=-0.18$ $\mathrm{N} \cdot \mathrm{m}, \quad F_{\theta_{2}}=-2.3 \quad \mathrm{~N} \cdot \mathrm{m} \quad$ y $\quad F_{\theta_{3}}=-1.2 \quad \mathrm{~N} \cdot \mathrm{m} . \quad \mathbf{M}_{b}$ es el momento de sujeción de la garra inferior, alrededor de los ejes Cartesianos del mundo W.

Para el segundo ejemplo, el robot se encuentra en la misma configuración, pero ahora la garra superior está fija y se tienen las siguientes velocidades articulares $(\mathrm{en} \mathrm{rad} / \mathrm{s}): \dot{\theta}_{0}=1, \dot{\theta}_{1}=0, \dot{\theta}_{2}=-2, \dot{\theta}_{3}=-3$. La resolución de la dinámica inversa proporciona las siguientes fuerzas y momentos: $F_{x_{a}}=7.06 \mathrm{~N}, F_{y_{a}}=-6.5$ $\mathrm{N}, F_{z_{a}}=35.9 \mathrm{~N}, \mathbf{M}_{a}=[6.1,-11,-3.9]^{T} \mathrm{~N} \cdot \mathrm{m}, F_{\theta_{0}}=-1.04$ $\mathrm{N} \cdot \mathrm{m}, F_{\theta_{1}}=1.56 \mathrm{~N} \cdot \mathrm{m}, F_{\theta_{2}}=3.44 \mathrm{~N} \cdot \mathrm{m}$ y $F_{\theta_{3}}=-0.45 \mathrm{~N} \cdot \mathrm{m}$. $\mathbf{M}_{a}$ es el momento de sujeción de la garra superior, alrededor de los ejes Cartesianos del mundo W.

En ambos ejemplos, los valores mostrados coinciden con los valores obtenidos en simulaciones realizadas en SimMechanics como comprobación.

\section{CONCLUSIÓN}

Este artículo ha presentado la obtención del modelo dinámico completo de un robot trepador de tipo pasoa-paso, con arquitectura serie y 4 grados de libertad. El modelo obtenido es totalmente general, siendo válido independientemente de cuál de las garras del robot se fije, e independientemente de la posición y orientación del robot en la estructura a explorar.

El modelo obtenido ha sido implementado en una intuitiva interfaz gráfica de usuario, que puede utilizarse para simular la cinemática y la dinámica inversa del robot en cualquier punto de una estructura. En el futuro se añadirán más funcionalidades a esta herramienta, así como más robots trepadores, lo que permitirá comparar distintas arquitecturas de robots trepadores en términos cinemáticos y de sus prestaciones dinámicas. 

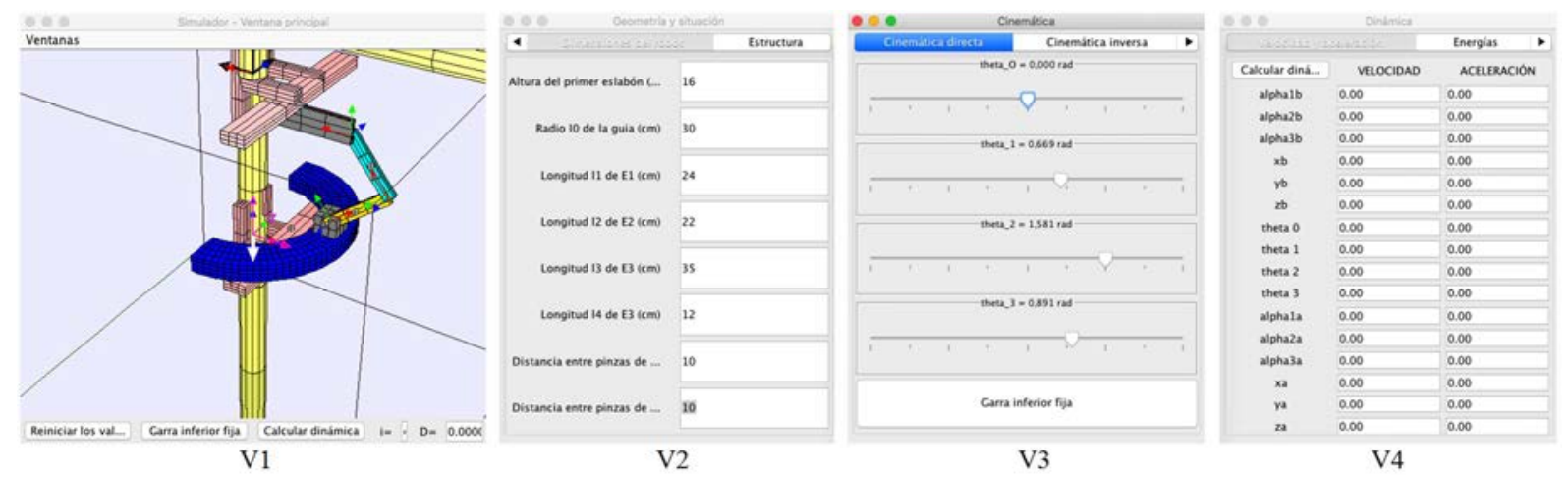

Figura 3: Herramienta desarrollada para simular el robot trepador

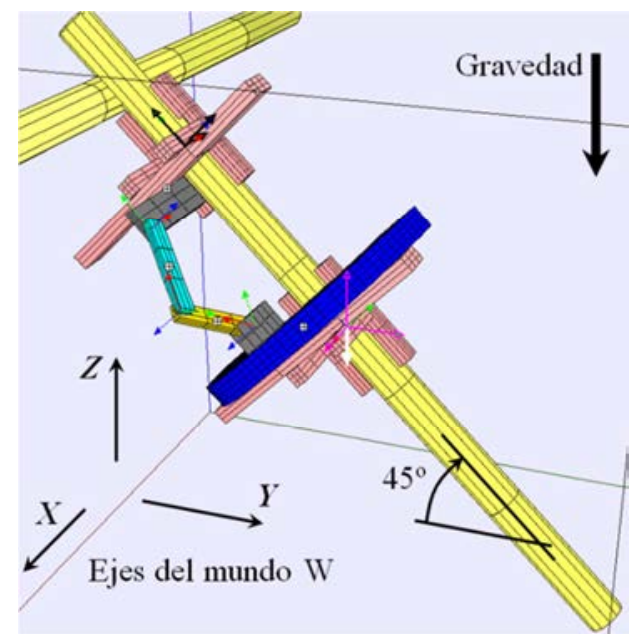

Figura 4: Configuración estudiada en los ejemplos

\section{Agradecimientos}

Este trabajo ha sido financiado por el Ministerio de Educación a través de la ayuda FPU13/00413, por el Ministerio de Economía a través del proyecto DPI2013-41557-P, y por la Generalitat Valenciana a través del proyecto AICO/2015/021.

\section{Referencias}

[1] Aracil R., Saltaren R.J., and Reinoso O. A climbing parallel robot: a robot to climb along tubular and metallic structures. IEEE Robotics \& Automation Magazine, 13(1):16-22, 2006.

[2] Balaguer C., Giménez A., Pastor J.M.,Padrón V.M., and Abderrahim M. A climbing autonomous robot for inspection applications in 3D complex environments. Robotica, 18(3):287-297, 2000.

[3] Figliolini G., Rea P., and Conte M. Mechanical Design of a Novel Biped Climbing and Walking Robot. In Parenti-Castelli V. and Schiehlen $W$., editors, ROMANSY 18 Robot Design, Dynamics and Control, pp. 199-206. Springer Vienna, 2010.

[4] Guan Y., Jiang L., Zhu H., Zhou X., Cai C., Wu W., Li Z., Zhang H., and Zhang X. Climbot: A modular bio-inspired biped climbing robot. In Proceedings of the 2011 IEEE/RSJ International Conference on Intelligent Robots and Systems, pp. 1473-1478, September 2011.

[5] Holm D.D., Schmah T., and Stoica C. Geometric Mechanics and Symmetry: From Finite to Infinite Dimensions. Oxford University Press, 2009.

[6] Peidro A., Gil A., Marin J.M., and Reinoso O. Inverse Kinematic Analysis of a Redundant Hybrid Climbing Robot. Int. J. Adv. Robot. Syst., 12:163, 2015.

[7] Shvalb N., Moshe B.B., and Medina O. A realtime motion planning algorithm for a hyperredundant set of mechanisms. Robotica, 31(8):1327-1335, 2013.

[8] Tavakoli M. Design, implementation, path planning and control of a pole climbing robot. $\mathrm{PhD}$ thesis, University of Coimbra, July 2010.

[9] Tavakoli M., Marques L., and De Almeida A.T. 3DCLIMBER: Climbing and manipulation over 3D structures. Mechatronics, 21(1):48-62, 2011.

[10] Tavakoli M., Zakerzadeh M.R., Vossoughi G.R., and Bagheri S. A hybrid pole climbing and manipulating robot with minimum DOFs for construction and service applications. Industrial Robot: An International Journal, 32(2):171-178, 2005.

[11] Tsai L.W. Robot Analysis: the mechanics of serial and parallel manipulators. John Wiley \& Sons, Inc., 1999. 